\title{
Thermophysical Properties of Binary Mixtures of Dimethylsulfoxide with 1-Phenylethanone and 1,4-Dimethylbenzene at Various Temperatures
}

\author{
Harmandeep Singh Gill and V. K. Rattan \\ Dr. SSB University Institute of Chemical Engineering and Technology, Panjab University, Chandigarh 160014, India \\ Correspondence should be addressed to Harmandeep Singh Gill; harman_gill@outlook.com
}

Received 18 September 2013; Revised 10 December 2013; Accepted 1 January 2014; Published 24 February 2014

Academic Editor: K. A. Antonopoulos

Copyright ( $\odot 2014$ H. S. Gill and V. K. Rattan. This is an open access article distributed under the Creative Commons Attribution License, which permits unrestricted use, distribution, and reproduction in any medium, provided the original work is properly cited.

\begin{abstract}
This research article reports the experimental results of the density, viscosity, refractive index, and speed of sound analysis of binary mixtures of dimethylsulfoxide (DMSO) + 1-phenylethanone (acetophenone) and + 1,4-dimethylbenzene (para-xylene) over the whole composition range at $313.15,318.15,323.15$, and $328.15 \mathrm{~K}$ and at atmospheric pressure. The excess molar volumes $\left(V^{E}\right)$, viscosity deviations $(\Delta \eta)$, excess Gibbs energy of activation $\left(G^{E}\right)$, deviations in isentropic compressibility $\left(K_{S}^{E}\right)$, deviations in speed of sound $\left(u^{E}\right)$, and deviations in the molar refraction $(\Delta R)$ were calculated from the experimental data. The computed quantities were fitted to the Redlich-Kister equation to derive the coefficients and estimate the standard error values. The viscosities have also been correlated with two, and three-parameter models, that is, Heric correlation, McAllister model, and Grunberg-Nissan correlation, respectively.
\end{abstract}

\section{Introduction}

This paper is a continuation of our ongoing research on the solution properties. Studies of the thermodynamic properties of binary mixtures play an important role in the fundamental understanding of different molecules and the interactions prevalent in them. In the present study, data on density, viscosity, refractive index, and speed of sound of binary mixtures of dimethylsulfoxide (DMSO) + 1-phenylethanone (acetophenone) and 1,4-Dimethylbenzene (para-xylene) at $313.15,318.15,323.15$, and $328.15 \mathrm{~K}$ has been measured experimentally. From these results, the excess molar volumes, viscosity deviations, deviations in molar refraction, deviations in speed of sound, and isentropic compressibility have been derived. Dimethylsulfoxide is a versatile nonaqueous dipolar aprotic solvent having wide range of applications like in veterinary medicine, dermatology, microbiology, experimental immunology, and enzyme catalyzed reactions. It can easily pass through membranes, a quality which has been verified by numerous researchers. It has the ability to penetrate through living tissues without damaging them.
Therefore an anesthetic or penicillin can be carried through the skin without using a needle which makes it paramount in medicinal field. Acetophenone is the simplest aromatic ketone organic compound. It can easily dissolve in water, but, since it is denser than water, it tends to sink. Its vapor is heavier than air and when inhaled in high concentrations it can be narcotic and also mild irritant to the eyes and skin. It is mostly used to create fragrances that smell like cherry, almond, strawberry, or other fruits. Acetophenone can also be found naturally occurring in fruits such as apple and banana. Para-xylene is an aromatic hydrocarbon based on benzene with two methyl substituents, opposite to each other. It is a colorless, flammable liquid and is insoluble in water. It is used as a thinner for paint and in paints and varnishes. The study of the thermodynamic properties of DMSO + 1phenylethanone (acetophenone) and + 1,4-dimethylbenzene (para-xylene) mixtures is of interest mainly in industrial fields where solvent mixtures could be used as selective solvents for numerous reactions. In principle, interactions between the molecules can be established from the study of the deviations from ideal behavior of physical properties 
TABLE 1: Physical properties of the components at $298.15 \mathrm{~K}$.

\begin{tabular}{|c|c|c|c|c|c|c|}
\hline \multirow{2}{*}{ Component } & \multicolumn{2}{|c|}{$\rho\left(\mathrm{g} \cdot \mathrm{cm}^{-3}\right)$} & \multicolumn{2}{|c|}{$\eta(\mathrm{mPa} \cdot \mathrm{s})$} & \multicolumn{2}{|c|}{$n_{D}$} \\
\hline & Exptl. & Lit. & Exptl. & Lit. & Exptl. & Lit. \\
\hline Acetophenone & 1.0283 & $1.0263^{17}$ & 0.01681 & $0.01681^{17}$ & 1.5050 & $1.5005^{17}$ \\
\hline DMSO & 1.0940 & 1.09537 & 1.9834 & 1.9910 & 1.4798 & 1.4775 \\
\hline p-Xylene & 8.5661 & $8.5662^{16}$ & 0.609 & $0.611^{16}$ & 1.4933 & $1.4933^{16}$ \\
\hline
\end{tabular}

such as molar volume and isentropic compressibility. The negative or positive deviations from the ideal value depend on the type and the extent of the interactions between the unlike molecules, as well as on the composition and the temperature. The variation of the isentropic compressibility is analogous of that of the excess molar volume, whereas the change of the deviation in speed of sound tends to become the inverse [1]. Physical and transport properties of liquid mixtures also affect most separation procedures, such as liquid-liquid extraction, gas absorption, and distillation [2]. The mixture DMSO- $p$-xylene has been earlier reported twice in literature at different temperatures $[1,3]$.

\section{Experimental Section}

2.1. Materials. The chemicals used are of analytical reagent grade. Dimethylsulfoxide (DMSO) is from Riedel, Germany; 1-phenylethanone (acetophenone) and 1,4-dimethylbenzene (para-xylene) are from S-D Fine Chemicals, Mumbai. The chemicals were purified using standard procedure [4] and were stored over molecular sieves. The purity of the chemicals was verified by comparing density, viscosity, and refractive index with the known values reported in the literature as shown in Table 1. All the compositions were prepared by using SARTORIUS balance. The possible uncertainty in the mole fraction is estimated to be less than $\pm 1 \times 10^{-4}$.

2.2. Viscosity. Kinematic viscosities were measured by using a calibrated modified Ubbelohde viscometer [5]. The calibration of viscometer was done at each temperature in order to determine the constants $A$ and $B$ of the following equation:

$$
v=\frac{\eta}{\rho}=A t+\frac{B}{t}
$$

The viscometer was kept vertically in a transparent-walled water bath with a thermal stability of $\pm 0.05 \mathrm{~K}$ for about 30 minutes to attain thermal equilibrium. Flow time was measured with an electronic stop watch with precision of $\pm 0.01 \mathrm{~s}$. The corresponding uncertainty in the kinematic viscosity is $\pm 0.001 \times 10^{-6} \mathrm{~m}^{2} \mathrm{~s}^{-1}$. The efflux time was repeated at least three times for each composition and the average of these readings was taken. The temperature of the bath was maintained constant with the help of a circulating type cryostat (type MK70, MLW, Germany). The dynamic viscosities were found out after the DSA analysis, that is, by dividing the above found kinematic viscosity by density. The uncertainty in the values of dynamic viscosity is within $\pm 0.003 \mathrm{mPa} \cdot \mathrm{s}$.
2.3. Density and Speed of Sound. Density and speed of sound were measured with the help of an ANTON PAAR density meter (DSA 5000). The accuracy in the measurement of density and speed of sound is $\pm 0.000005 \mathrm{~g} \mathrm{~cm}^{-3}$ and $\pm 0.5 \mathrm{~ms}^{-1}$, respectively. The density meter was calibrated by using triply distilled degassed water.

2.4. Refractive Index. Refractive indices were measured for sodium D-line by ABBE-3L refractometer having Bausch and Lomb lenses. The temperature was maintained constant with the help of water bath used for the viscosity measurement. A minimum of three independent readings were taken for each composition, and the average value was considered in all the calculations. Refractive index values are accurate up to \pm 0.0001 units.

\section{Experimental Results and Correlations}

At least three independent readings of all the physical property measurements of density $(\rho)$, viscosity $(\eta)$, refractive index $\left(n_{D}\right)$, and speed of sound $(u)$ were taken for each composition and the averages of these experimental values are presented in Tables 2 and 3 for both systems. The experimentally determined values are used for the deviation calculations.

3.1. Excess Molar Volume. Density values are used to evaluate excess molar volume by the equation

$$
V^{E}=\frac{x_{1} M_{1}+x_{2} M_{2}}{\rho}-\frac{x_{1} M_{1}}{\rho_{1}}-\frac{x_{2} M_{2}}{\rho_{2}},
$$

where $\rho_{1}, \rho_{2}$ are the densities of pure components and $\rho$ is the density of the mixture. $M_{1}, M_{2}$ are the molar mass of the two components and $x_{1}, x_{2}$ are the mole fraction of DMSO.

Excess Gibbs' free energy of activation has been also calculated using the viscosity and density of the mixture by the equation

$$
\Delta G^{E}=R T\left[\ln (\eta V)-\sum_{i=1}^{2} x_{i} \ln \left(\eta_{i} V_{i}\right)\right],
$$

where $R$ is a universal gas constant, $T$ is the temperature of the mixture, and $\eta$ and $\eta_{i}$ are the viscosities of the mixture and pure compound, respectively. $V, V_{i}$ refer to the molar volume of the mixture and pure components, respectively.

3.2. Viscosity Calculations. The deviation in viscosity is obtained by the following equation:

$$
\Delta \eta=\eta-\eta_{1} x_{1}-\eta_{2} x_{2}
$$


TABLE 2: Refractive indices, $n_{D}$, density, $\rho$, speed of sound, $u$, and viscosity, $\eta$, for DMSO(1) + acetophenone(2) system at different temperatures.

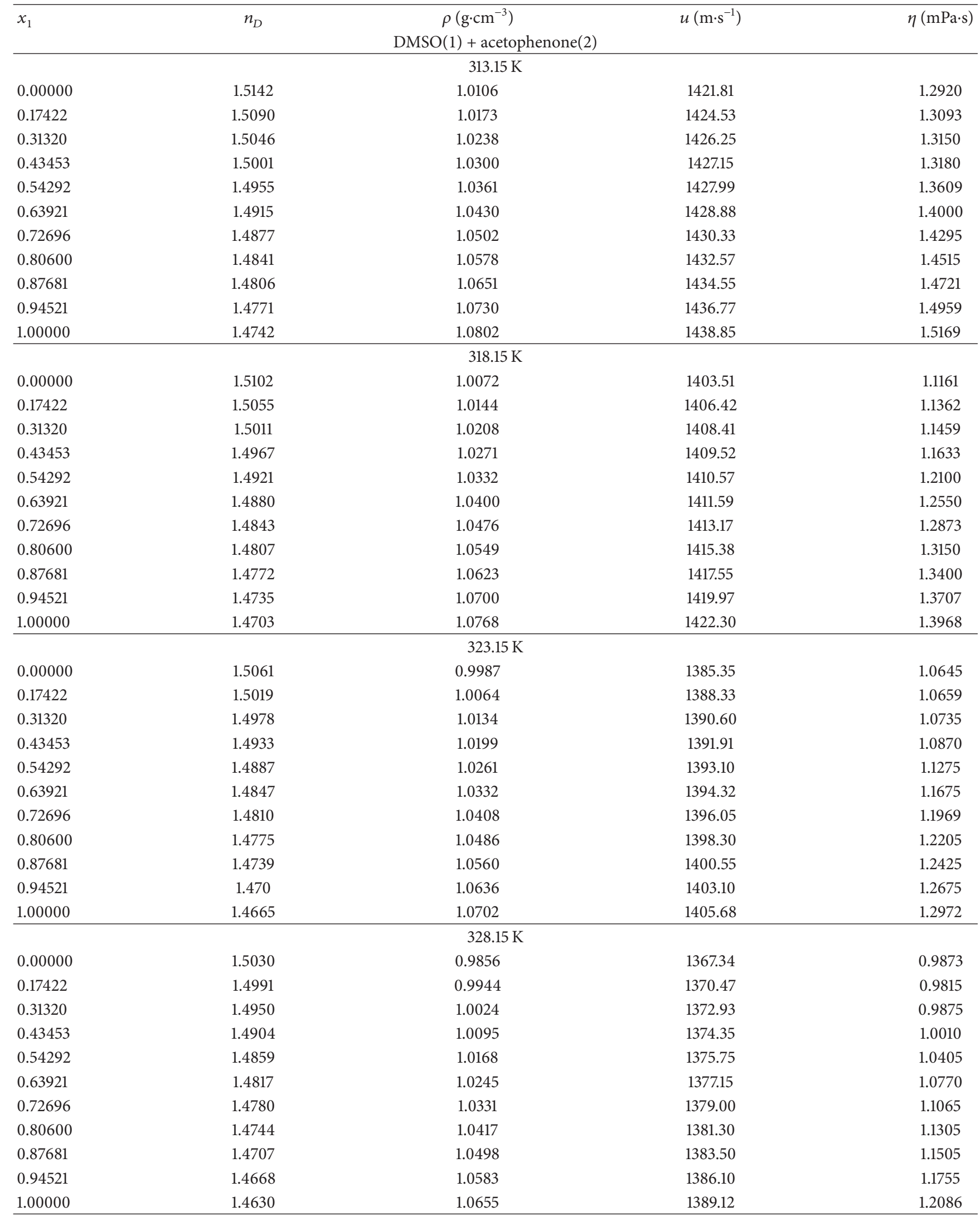


TABLE 3: Refractive indices, $n_{D}$, density, $\rho$, speed of sound, $u$, and viscosity, $\eta$, for DMSO(1) + p-xylene(2) system at different temperatures.

\begin{tabular}{|c|c|c|c|c|}
\hline$x_{1}$ & $n_{D}$ & $\begin{array}{c}\rho\left(\mathrm{g} \cdot \mathrm{cm}^{-3}\right) \\
\mathrm{DMSO}(1)+\mathrm{p} \text {-xylene }(2)\end{array}$ & $u\left(\mathrm{~m} \cdot \mathrm{s}^{-1}\right)$ & $\eta(\mathrm{mPa} \cdot \mathrm{s})$ \\
\hline \multicolumn{5}{|c|}{$313.15 \mathrm{~K}$} \\
\hline 0.00000 & 1.4850 & 0.8445 & 1247.96 & 0.5175 \\
\hline 0.15820 & 1.4843 & 0.8720 & 1264.82 & 0.5979 \\
\hline 0.30056 & 1.4839 & 0.8999 & 1281.64 & 0.6777 \\
\hline 0.42694 & 1.4828 & 0.9293 & 1297.89 & 0.7389 \\
\hline 0.52448 & 1.4813 & 0.9577 & 1310.14 & 0.8106 \\
\hline 0.62739 & 1.4790 & 0.9923 & 1325.23 & 0.8875 \\
\hline 0.72506 & 1.4778 & 1.0233 & 1346.79 & 0.9889 \\
\hline 0.80799 & 1.4765 & 1.0477 & 1373.38 & 1.0997 \\
\hline 0.87462 & 1.4747 & 1.0557 & 1395.79 & 1.2275 \\
\hline 0.94123 & 1.4727 & 1.0723 & 1418.20 & 1.3757 \\
\hline 1.00000 & 1.4717 & 1.0802 & 1439.15 & 1.5116 \\
\hline \multicolumn{5}{|c|}{$318.15 \mathrm{~K}$} \\
\hline 0.00000 & 1.4831 & 0.8395 & 1227.99 & 0.4923 \\
\hline 0.15820 & 1.4823 & 0.8665 & 1246.38 & 0.5675 \\
\hline 0.30056 & 1.4816 & 0.8942 & 1263.94 & 0.6422 \\
\hline 0.42694 & 1.4803 & 0.9233 & 1281.67 & 0.6971 \\
\hline 0.52448 & 1.4789 & 0.9517 & 1295.39 & 0.7555 \\
\hline 0.62739 & 1.4767 & 0.9870 & 1310.77 & 0.8179 \\
\hline 0.72506 & 1.4754 & 1.0183 & 1331.90 & 0.9090 \\
\hline 0.80799 & 1.4737 & 1.0417 & 1358.39 & 1.0220 \\
\hline 0.87462 & 1.4719 & 1.0497 & 1380.68 & 1.1401 \\
\hline 0.94123 & 1.4701 & 1.0668 & 1404.05 & 1.2705 \\
\hline 1.00000 & 1.4690 & 1.0768 & 1423.09 & 1.3822 \\
\hline \multicolumn{5}{|c|}{$323.15 \mathrm{~K}$} \\
\hline 0.00000 & 1.4812 & 0.8348 & 1207.94 & 0.4603 \\
\hline 0.15820 & 1.4802 & 0.8610 & 1226.95 & 0.5401 \\
\hline 0.30056 & 1.4794 & 0.8882 & 1245.22 & 0.6030 \\
\hline 0.42694 & 1.4777 & 0.9167 & 1263.54 & 0.6550 \\
\hline 0.52448 & 1.4761 & 0.9443 & 1277.98 & 0.7107 \\
\hline 0.62739 & 1.4744 & 0.9804 & 1292.79 & 0.7697 \\
\hline 0.72506 & 1.4727 & 1.0110 & 1314.76 & 0.8557 \\
\hline 0.80799 & 1.4710 & 1.0333 & 1340.33 & 0.9776 \\
\hline 0.87462 & 1.4690 & 1.0410 & 1363.23 & 1.0895 \\
\hline 0.94123 & 1.4675 & 1.0590 & 1385.97 & 1.1999 \\
\hline 1.00000 & 1.4665 & 1.0702 & 1404.14 & 1.2972 \\
\hline \multicolumn{5}{|c|}{$328.15 \mathrm{~K}$} \\
\hline 0.00000 & 1.4793 & 0.8298 & 1188.15 & 0.4385 \\
\hline 0.15820 & 1.4781 & 0.8553 & 1208.59 & 0.5150 \\
\hline 0.30056 & 1.4771 & 0.8823 & 1227.53 & 0.5707 \\
\hline 0.42694 & 1.4754 & 0.9106 & 1247.59 & 0.6092 \\
\hline 0.52448 & 1.4736 & 0.9377 & 1262.23 & 0.6579 \\
\hline 0.62739 & 1.4715 & 0.9733 & 1278.99 & 0.7175 \\
\hline 0.72506 & 1.4699 & 1.0045 & 1300.23 & 0.7917 \\
\hline 0.80799 & 1.4683 & 1.0261 & 1325.12 & 0.9158 \\
\hline 0.87462 & 1.4663 & 1.0341 & 1348.21 & 1.0109 \\
\hline 0.94123 & 1.4652 & 1.0530 & 1370.98 & 1.1152 \\
\hline 1.00000 & 1.4640 & 1.0655 & 1388.57 & 1.1974 \\
\hline
\end{tabular}


where $\eta$ is the viscosity of mixture and $\eta_{1}, \eta_{2}$ refer to the viscosities of pure components.

McAllister [6] model

$$
\begin{aligned}
\ln v= & x_{1}^{3} \ln v_{1}+x_{2}^{3} \ln v_{2}+3 x_{1}^{2} x_{2} \ln \eta_{12}+3 x_{1} x_{2}^{2} \ln \eta_{21} \\
& -\ln \left[x_{1}+x_{2} \frac{M_{2}}{M_{1}}\right]+3 x_{1}^{2} x_{2} \ln \left[\frac{2+M_{2} / M_{1}}{3}\right] \\
& +3 x_{1} x_{2}^{2} \ln \left[\frac{1+\left(2 M_{2} / M_{1}\right)}{3}\right]+x_{2}^{3} \ln \left[\frac{M_{2}}{M_{1}}\right] .
\end{aligned}
$$

Herric [6] correlation

$$
\begin{aligned}
\ln v= & x_{1} \ln v_{1}+x_{2} \ln v_{2}+x_{1} x_{2}\left[\alpha_{12}+\alpha_{12}\left(x_{1}-x_{2}\right)\right] \\
& -\ln M_{\text {mix }}+x_{1} \ln M_{1}+x_{2} \ln M_{2}
\end{aligned}
$$

and Grunberg-Nissan [7] equation

$$
\ln (\eta)=x_{1} \ln \left(\eta_{1}\right)+x_{2} \ln \left(\eta_{2}\right)+d_{12} x_{1} x_{2}
$$

have been fitted to viscosity data and it was found that both have the same standard errors at each temperature.

3.3. Isentropic Compressibility. The experimental results for the speed of sound of binary mixtures are listed in Tables 2 and 3. The isentropic compressibility was evaluated by using $K_{S}=u^{-2} \rho^{-1}$ and the deviation in isentropic compressibility is calculated using the following equation:

$$
K_{S}^{E}=K_{S}-K_{S}^{\mathrm{id}}
$$

where $K_{S}^{\mathrm{id}}$ stands for isentropic compressibility for an ideal mixture calculated using Benson-Kiyohara model $[8,9]$ :

$$
\begin{aligned}
K_{S}^{\mathrm{id}}= & \sum_{i=1}^{2} \Phi_{i}\left[K_{S, i}+\frac{T V_{i}\left(\alpha_{i}^{2}\right)}{C_{p, i}}\right] \\
& -\left\{\frac{T\left(\sum_{i=1}^{2} x_{i} V_{i}\right)\left(\sum_{i=1}^{2} \Phi_{i} a_{i}\right)^{2}}{\sum_{i=1}^{2} x_{i} C_{p, i}}\right\},
\end{aligned}
$$

where $a_{i}$ and $C_{p}$ are the thermal expansion coefficient and molar heat capacity of the $i$ th components, respectively.

The deviation in speed of sound is given by

$$
\Delta u=u-x_{1} u_{1}-x_{2} u_{2} \text {. }
$$

3.4. Molar Refraction. Refractive indices have been used for the calculation of molar refraction $\left(R_{m}\right)$ that is obtained by using Lorentz-Lorenz equation [8].

Deviation in molar refraction $(\Delta R)$ is calculated by the following equation:

$$
\begin{gathered}
R=R_{m}-\sum \Phi_{i} R_{i}, \\
\Phi_{i}=\frac{x_{i}}{\sum x_{j} V_{j}},
\end{gathered}
$$

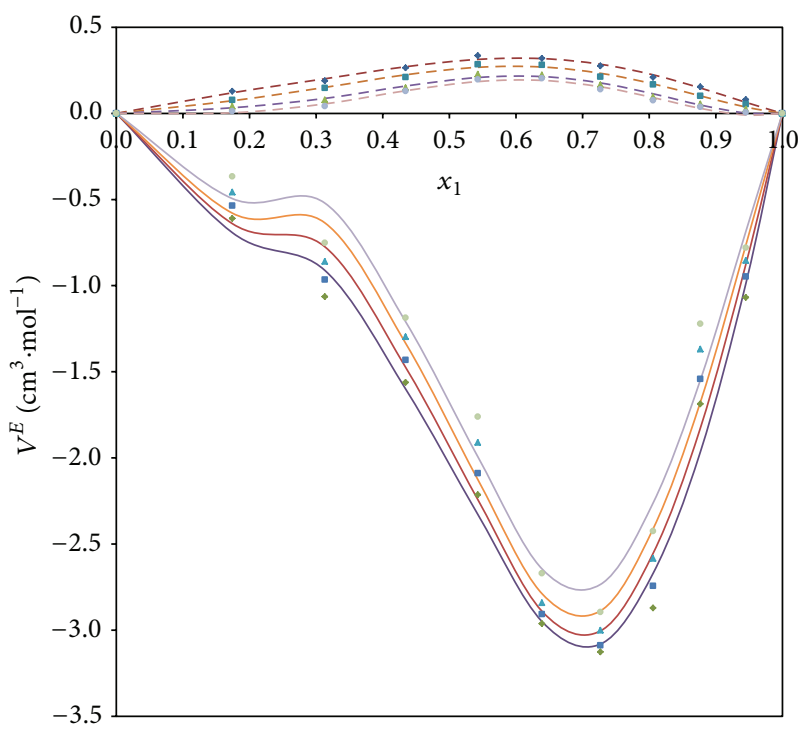

Figure 1: Experimental and calculated excess molar volume for (i) DMSO(1) + acetophenone(2) and (ii) $\mathrm{DMSO}(1)+\mathrm{p}$-Xylene(2) at $313.15 \mathrm{~K}, \diamond, 318.15 \mathrm{~K}, \mathbf{\square}, 323.15 \mathrm{~K}, \boldsymbol{\Delta}, 328.15 \mathrm{~K}$, •; symbols represent the experimental values; dotted lines represent DMSOacetophenone mixture and solid lines represent DMSO-p-Xylene mixture, both optimised by Redlich-Kister parameters.

where $n_{D}$ refers to the refractive index, $R_{m}$ is molar refraction of the mixture, $R_{i}$ is molar refraction of the $i$ th component, and $\Phi$ is ideal state volume fraction.

All the deviations $\left(V^{E}, \Delta R, \Delta \eta, \Delta u\right.$, and $\left.K_{S}^{E}\right)$ have been fitted to Redlich-Kister polynomial regression of the type

$$
\Delta Y=x_{1} x_{2} \sum_{i=1}^{m} A_{i}\left(1-2 x_{1}\right)^{i-1}
$$

to derive the constant $A_{i}$ using the method of the least square.

Standard deviation for each case is calculated by

$$
\sigma=\left[\frac{\sum\left(\Delta Y_{\mathrm{exptl}}-\Delta Y_{\mathrm{calcd}}\right)^{2}}{m-n}\right]^{0.5},
$$

where $m$ is the number of data points and $n$ is the number of coefficients. Derived parameters of the Redlich-Kister equation (12) and standard deviations (13) are presented in Tables 4 and 5.

\section{Discussions}

The excess molar volume from 313.15 to $328.15 \mathrm{~K}$ versus the mole fraction of both mixtures with respect to DMSO is shown in Figure 1. The molar volume of the mixtures and the viscosity data have been used for the calculation of Gibbs' free energy presented in Figure 5. The $V^{E}$ values decrease with increasing temperatures for the systems but are positive in case of DMSO-acetophenone mixture and negative for DMSO-p-xylene mixture. Treszczanowicz et al. 
TABLE 4: Derived parameters of Redlich-Kister equation (12) and standard deviation (13) for various functions of the binary mixtures at different temperatures (DMSO-acetophenone).

\begin{tabular}{|c|c|c|c|c|c|}
\hline$T / K$ & $A_{0}$ & $A_{1}$ & $A_{2}$ & $A_{3}$ & $\sigma$ \\
\hline \multicolumn{6}{|c|}{$V^{E}\left(\mathrm{~cm}^{3} \cdot \mathrm{mol}^{-1}\right)$} \\
\hline 313.15 & 1.2005 & 0.741 & -0.1784 & -0.6838 & 0.01377 \\
\hline 318.15 & 1.0176 & 0.7493 & -0.5481 & -0.7711 & 0.01379 \\
\hline 323.15 & 0.7709 & 0.8700 & -0.7689 & -1.2620 & 0.01527 \\
\hline 328.15 & 0.6714 & 0.9374 & -0.9528 & -1.3257 & 0.01381 \\
\hline \multicolumn{6}{|c|}{$\Delta \eta(\mathrm{mPa} \cdot \mathrm{s})$} \\
\hline 313.15 & -0.2371 & 0.2135 & 0.2487 & -0.4972 & 0.00461 \\
\hline 318.15 & -0.2575 & 0.2259 & 0.1846 & -0.4935 & 0.00323 \\
\hline 323.15 & -0.2722 & 0.2375 & 0.0974 & -0.4693 & 0.00309 \\
\hline 328.15 & -0.2887 & 0.2407 & 0.0508 & -0.4755 & 0.00290 \\
\hline \multicolumn{6}{|c|}{$K_{s}^{E}\left(\mathrm{TPa}^{-1}\right)$} \\
\hline 313.15 & 4.8515 & 15.4271 & -1.3121 & -12.5359 & 0.11076 \\
\hline 318.15 & 3.5099 & 15.7048 & -1.8315 & -12.902 & 0.09236 \\
\hline 323.15 & 2.0047 & 21.1502 & -4.7008 & -34.8976 & 0.44672 \\
\hline 328.15 & 0.0146 & 15.1308 & -2.0538 & -11.8737 & 0.17247 \\
\hline \multicolumn{6}{|c|}{$\Delta R$} \\
\hline 313.15 & 0.06817 & 0.039577 & -0.06186 & -0.09945 & 0.006271 \\
\hline 318.15 & 0.102475 & 0.056959 & -0.00441 & -0.06411 & 0.003073852 \\
\hline 323.15 & 0.147987 & 0.106458 & 0.080891 & -0.10664 & 0.005976 \\
\hline 328.15 & 0.175329 & 0.134545 & 0.138756 & -0.14317 & 0.008927 \\
\hline \multicolumn{6}{|c|}{$\Delta G^{E}\left(\mathrm{~J} \cdot \mathrm{mol}^{-1}\right)$} \\
\hline 313.15 & -66.3882 & 516.3512 & 492.0794 & -981.8602 & 9.00998 \\
\hline 318.15 & -141.309 & 625.565 & 410.846 & -1119.199 & 7.38255 \\
\hline 323.15 & -234.912 & 700.1368 & 230.6251 & -1143.3850 & 7.33602 \\
\hline 328.15 & -321.946 & 780.1317 & 132.3463 & -1256.91 & 7.21134 \\
\hline
\end{tabular}

[10] and Roux and Desnoyers [11] suggested that $V^{E}$ is the resultant contribution from several opposing effects. These effects can be primarily divided into three types, namely, chemical, physical, and structural. A physical contribution, that is, specific interactions between the real species present in the mixture, contribute in negative terms to $V^{E}$. The chemical or specific intermolecular interactions result in a volume decrease, and these include charge transfer type forces and other complex forming interactions. This effect also contributes in negative values to $V^{E}$. The structural contributions are mostly negative and can arise from several effects, especially from changes of free volume and interstitial accommodation. In other words, structural contributions arising from geometrical fitting of one component into the other due to the differences in the free volume and molar volume between components lead to a negative contribution to $V^{E}$. The viscosity and deviations are presented in Table 2 and plotted in Figure 2, respectively, for both systems. The viscosity deviations decrease with the increase in temperature for both systems. The negative $\Delta \eta$ values are generally observed for systems where dispersion or weak dipole-dipole forces are primarily responsible for interaction between the component molecules. The viscosity data is also fitted to the two, and the three-parameter models, that is, Herric correlation, the McAllister model, and Grunberg-Nissan correlation, and the evaluated parameters are presented in Tables 6 and 7. The deviations in molar refraction for both systems are shown in Figure 3. The $\Delta R$ values are positive for acetophenone system for the whole composition range which goes on increasing as the temperature of the solution increases. The $\Delta R$ values are negative for para-xylene system for the whole composition range which goes on decreasing as the temperature of the solution increases. In general, the negative values of $\Delta R$ suggest that we have weak interactions between the component molecules in the mixture. The results of excess isentropic compressibility $\left(K_{S}^{E}\right)$ are also plotted in Figure 4 . The deviations for DMSO-acetophenone system are initially negative and then become positive when mole fraction is around 0.5 , whereas for DMSO- $p$-xylene system they are negative over the entire composition range. Deviation in Gibbs free energy for DMSO-acetophenone system follows an arbitrary path, going from negative to positive and vice versa twice, while for DMSO-para-xylene system the deviations are negative and increase with increasing temperature. 
TABLE 5: Derived parameters of Redlich-Kister equation (12) and standard deviation (13) for various functions of the binary mixtures at different temperatures (DMSO- $p$-xylene).

\begin{tabular}{|c|c|c|c|c|c|}
\hline$T / K$ & $A_{0}$ & $A_{1}$ & $A_{2}$ & $A_{3}$ & $\sigma$ \\
\hline \multicolumn{6}{|c|}{$V^{E}\left(\mathrm{~cm}^{3} \cdot \mathrm{mol}^{-1}\right)$} \\
\hline 313.15 & -8.5850 & -15.1234 & -6.084 & 12.8652 & 0.15687 \\
\hline 318.15 & -8.2010 & -15.9715 & -5.4345 & 15.6022 & 0.17024 \\
\hline 323.15 & -7.7110 & -16.483 & -4.7908 & 17.7396 & 0.19337 \\
\hline 328.15 & -7.1898 & -16.3123 & -4.0863 & 18.0512 & 0.20482 \\
\hline \multicolumn{6}{|c|}{$\Delta \eta(\mathrm{mPa} \cdot \mathrm{s})$} \\
\hline 313.15 & -0.9028 & -0.7268 & -0.2127 & 0.25 & 0.00553 \\
\hline 318.15 & -0.8069 & -0.7852 & -0.1061 & 0.5942 & 0.00475 \\
\hline 323.15 & -0.7589 & -0.7364 & 0.093 & 0.6582 & 0.00619 \\
\hline 328.15 & -0.7126 & -0.6943 & 0.2137 & 0.6476 & 0.0067 \\
\hline \multicolumn{6}{|c|}{$K_{s}^{E}\left(\mathrm{TPa}^{-1}\right)$} \\
\hline 313.15 & -119.0039 & -64.4234 & -74.9361 & -36.2431 & 1.03835 \\
\hline 318.15 & -137.2976 & -76.3846 & -70.5856 & -21.5844 & 0.97745 \\
\hline 323.15 & -148.0003 & -81.8649 & -67.7606 & -17.6011 & 0.84832 \\
\hline 328.15 & -166.5237 & -92.1709 & -61.6439 & -2.9525 & 0.87227 \\
\hline \multicolumn{6}{|c|}{$\Delta R$} \\
\hline 313.15 & -3.25811 & 4.787247 & -1.77213 & -3.9986 & 0.226222 \\
\hline 318.15 & -3.20078 & 4.955419 & -1.49922 & -4.5735 & 0.239049 \\
\hline 323.15 & -3.14001 & 5.033874 & -1.26969 & -4.89488 & 0.245337 \\
\hline 328.15 & -3.0793 & 5.147192 & -1.01287 & -5.28097 & 0.254675 \\
\hline \multicolumn{6}{|c|}{$\Delta G^{E}\left(\mathrm{~J} \cdot \mathrm{mol}^{-1}\right)$} \\
\hline 313.15 & -1022.2560 & -1753.98 & -140.348 & 1173.282 & 15.8755 \\
\hline 318.15 & -1013.8590 & -2190.4 & 88.3584 & 2252.137 & 14.30563 \\
\hline 323.15 & -1011.6820 & -2200.58 & 769.1765 & 2310.619 & 22.20041 \\
\hline 328.15 & -1113.8360 & -2249.67 & 1294.863 & 2307.221 & 24.1139 \\
\hline
\end{tabular}

TABLE 6: Interaction parameters for the McAllister model (5), Herric correlation (6), and Grunberg-Nissan correlation (7) for viscosity at different temperatures (DMSO-acetophenone).

\begin{tabular}{|c|c|c|c|}
\hline \multicolumn{4}{|c|}{ McAllister model } \\
\hline$T / K$ & $\eta_{12}$ & $\eta_{21}$ & $\sigma(\eta) / \mathrm{mPa} \cdot \mathrm{s}$ \\
\hline 313.15 & 1.3242 & 1.3473 & 0.00016 \\
\hline 318.15 & 1.350624 & 1.334886 & 0.00025 \\
\hline 323.15 & 1.377883 & 1.314174 & 0.00038 \\
\hline 328.15 & 1.348994 & 1.240874 & 0.00042 \\
\hline \multicolumn{4}{|c|}{ Herric correlation } \\
\hline$T / K$ & $\alpha_{12}$ & $\alpha_{12}$ & $\sigma(\eta) / \mathrm{mPa} \cdot \mathrm{s}$ \\
\hline 313.15 & -0.02338 & -0.01368 & 0.00016 \\
\hline 318.15 & -0.026 & -0.01677 & 0.00025 \\
\hline 323.15 & -0.02874 & -0.01993 & 0.00038 \\
\hline 328.15 & -0.03047 & -0.02084 & 0.00042 \\
\hline \multicolumn{4}{|c|}{ Grunberg-Nissan correlation } \\
\hline$T / K$ & \multicolumn{2}{|c|}{$d_{12}$} & $\sigma(\eta) / \mathrm{mPa} \cdot \mathrm{s}$ \\
\hline 313.15 & \multicolumn{2}{|c|}{-0.0956181} & 0.156414 \\
\hline 318.15 & \multicolumn{2}{|c|}{-0.1201454} & 0.095161 \\
\hline 323.15 & \multicolumn{2}{|c|}{-0.1618181} & 0.124392 \\
\hline 328.15 & \multicolumn{2}{|c|}{-0.1988545} & 0.2077 \\
\hline
\end{tabular}


TABLE 7: Interaction parameters for the McAllister model (5), Herric correlation (6), and Grunberg-Nissan correlation (7) for viscosity at different temperatures (DMSO- $p$-xylene).

\begin{tabular}{|c|c|c|c|}
\hline \multicolumn{4}{|c|}{ McAllister model } \\
\hline$T / K$ & $\eta_{12}$ & $\eta_{21}$ & $\sigma(\eta) / \mathrm{mPa} \cdot \mathrm{s}$ \\
\hline 313.15 & 1.04511700 & 0.88014850 & 0.00304 \\
\hline 318.15 & 1.03668800 & 0.87514410 & 0.00310 \\
\hline 323.15 & 1.02613100 & 0.87012170 & 0.00325 \\
\hline 328.15 & 1.01597400 & 0.86517980 & 0.00327 \\
\hline \multicolumn{4}{|c|}{ Herric correlation } \\
\hline$T / K$ & $\alpha_{12}$ & $\dot{\alpha}_{12}$ & $\sigma(\eta) / \mathrm{mPa} \cdot \mathrm{s}$ \\
\hline 313.15 & 0.04364338 & 0.13563440 & 0.00304 \\
\hline 318.15 & 0.03664824 & 0.13067020 & 0.00310 \\
\hline 323.15 & 0.03026920 & 0.12422880 & 0.00325 \\
\hline 328.15 & 0.02245963 & 0.11706220 & 0.00327 \\
\hline \multicolumn{4}{|c|}{ Grunberg-Nissan correlation } \\
\hline$T / K$ & \multicolumn{2}{|c|}{$d_{12}$} & $\sigma(\eta) / \mathrm{mPa} \cdot \mathrm{s}$ \\
\hline 313.15 & \multicolumn{2}{|c|}{-0.408281} & 0.01591 \\
\hline 318.15 & \multicolumn{2}{|c|}{-0.377472} & 0.025161 \\
\hline 323.15 & \multicolumn{2}{|c|}{-0.320472} & 0.01239 \\
\hline 328.15 & \multicolumn{2}{|c|}{-0.312945} & 0.02016 \\
\hline
\end{tabular}

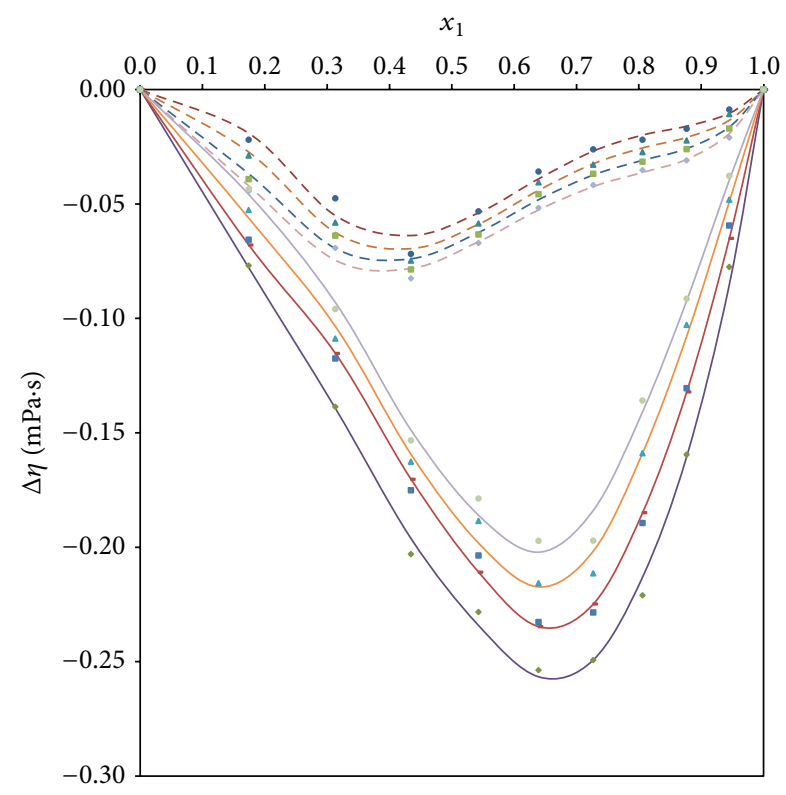

FIGURE 2: Experimental and calculated deviations in viscosity for (i) DMSO(1) + acetophenone(2) and (ii) $\mathrm{DMSO}(1)+\mathrm{p}$ xylene (2) at $313.15 \mathrm{~K}, \uparrow, 318.15 \mathrm{~K}, \mathbf{\varpi}, 323.15 \mathrm{~K}, \mathbf{\Delta}, 328.15 \mathrm{~K}$, ๑; symbols represent the experimental values; dotted lines represent DMSOacetophenone mixture and solid lines represent DMSO-p-xylene mixture, both optimised by Redlich-Kister parameters.

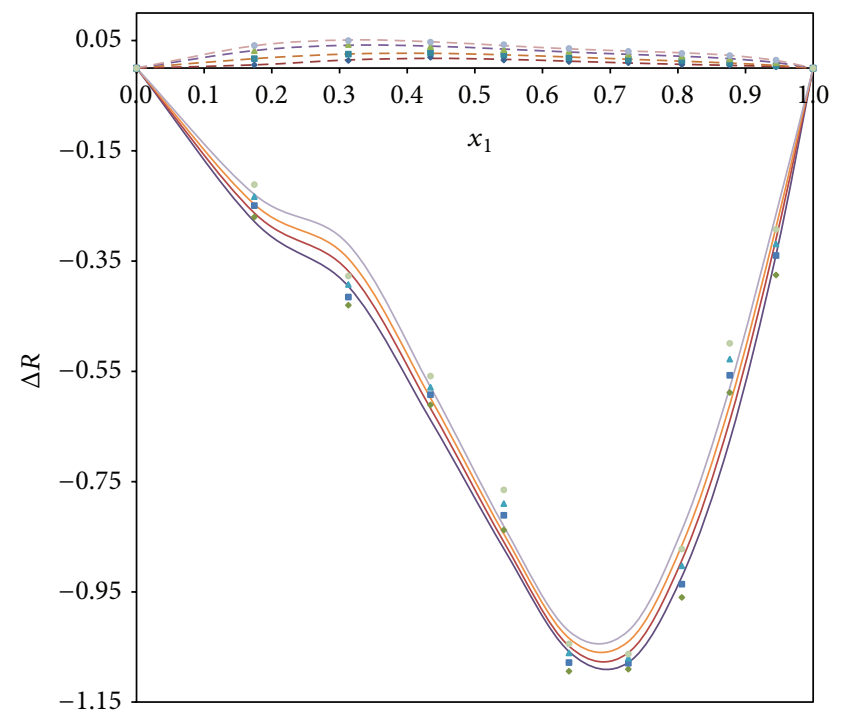

FIgURE 3: Experimental and calculated deviations in molar refraction for (i) $\mathrm{DMSO}(1)+$ acetophenone(2) and (ii) $\mathrm{DMSO}(1)+\mathrm{p}$ xylene $(2)$ at $313.15 \mathrm{~K}, \diamond, 318.15 \mathrm{~K}, \mathbf{\square}, 323.15 \mathrm{~K}, \boldsymbol{\Delta}, 328.15 \mathrm{~K}$, ๑; and symbols represent the experimental values; dotted lines represent DMSO-acetophenone mixture and solid lines represent DMSO-pxylene mixture, both optimised by Redlich-Kister parameters.

\section{Symbols Used}

$A_{1}, A_{2}, A_{3}, A_{4}$ : Parameters of Redlich-Kister equation $A_{12}, A_{21}: \quad$ Interaction coefficients of McAllister $\alpha_{12}, \dot{\alpha}_{12}: \quad$ Coefficients of Herric's correlation $\nu: \quad \quad$ Kinematic viscosity $\left(\mathrm{m}^{2} \mathrm{~s}^{-1}\right)$ $\rho: \quad$ Density $\left(\mathrm{g} \mathrm{cm}^{-3}\right)$ $\sigma: \quad$ Standard deviation

$\alpha: \quad$ Thermal expansion coefficient $\left(\mathrm{K}^{-1}\right)$

$\eta: \quad$ Dynamic viscosity $(\mathrm{mPa} \cdot \mathrm{s})$

$\Delta G^{E}:$ Excess Gibbs free energy $\left(\mathrm{Jmol}^{-1}\right)$

$V^{E}$ : Excess molar volume $\left(\mathrm{m}^{3} \mathrm{~mol}^{-1}\right)$

$\Delta K_{S}^{E}$ : Excess isentropic compressibility $\left(\mathrm{TPa}^{-1}\right)$ 


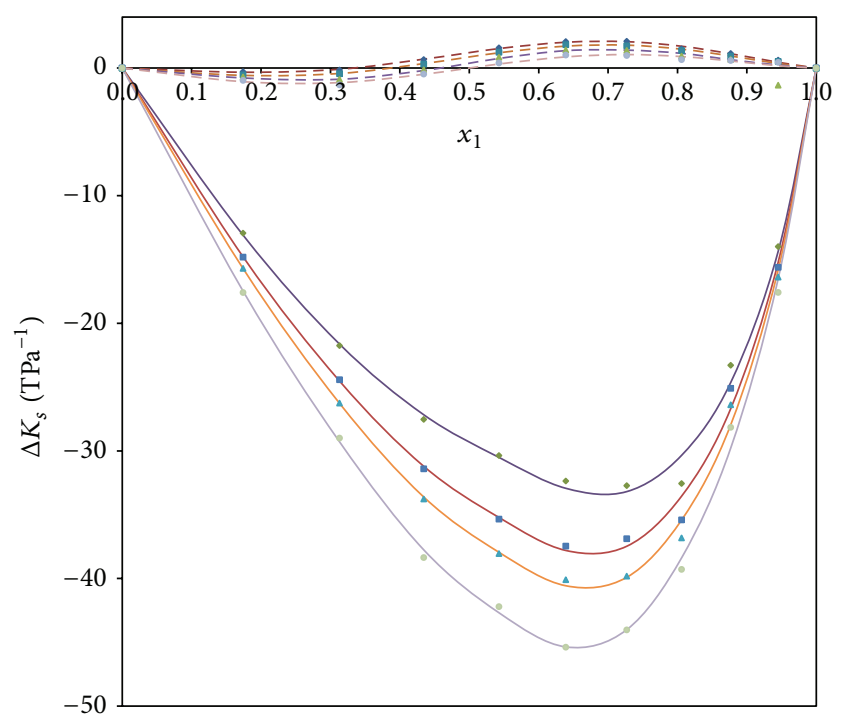

FIGURE 4: Experimental and calculated deviations in isentropic compressibility for (i) DMSO(1) + acetophenone(2) and (ii) $\operatorname{DMSO}(1)+$ p-xylene(2) at $313.15 \mathrm{~K}, \boldsymbol{}, 318.15 \mathrm{~K}, \mathbf{\square}, 323.15 \mathrm{~K}, \boldsymbol{\Delta}$, $328.15 \mathrm{~K}$, lines represent DMSO-Acetophenone mixture and solid lines represent DMSO- $p$-xylene mixture, both optimised by Redlich-Kister parameters.

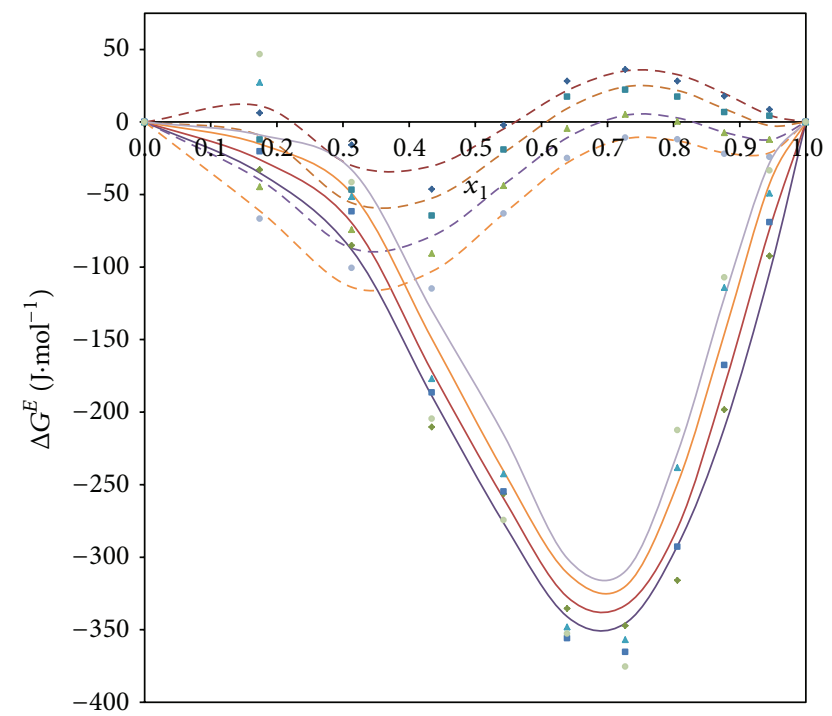

FIgURE 5: Experimental and calculated deviations in Gibbs free energy of activation for (i) $\mathrm{DMSO}(1)+$ acetophenone(2) and (ii) $\operatorname{DMSO}(1)+$ p-xylene(2) at $313.15 \mathrm{~K}, \boldsymbol{\diamond}, 318.15 \mathrm{~K}, \mathbf{\square}, 323.15 \mathrm{~K}, \boldsymbol{\Delta}$, $328.15 \mathrm{~K}, \bullet$; and symbols represent the experimental values; solid lines represent DMSO-Acetophenone mixture and dotted lines represent DMSO- $p$-xylene mixture, both optimised by RedlichKister parameters.

$R$ : Universal gas constant $\left(8.314 \mathrm{Jmol}^{-1} \mathrm{~K}^{-1}\right)$

$T$ : Absolute temperature (K)

$d_{12}$ : Grunberg-nissan parameter

$\Phi_{i}$ : Volume fraction (dimensionless).

\section{Conflict of Interests}

The authors declare that there is no conflict of interests regarding the publication of this paper.

\section{References}

[1] M. M. Palaiologou, G. K. Arianas, and N. G. Tsierkezos, “Thermodynamic investigation of dimethyl sulfoxide binary mixtures at 293.15 and 313.15 K," Journal of Solution Chemistry, vol. 35, no. 11, pp. 1551-1565, 2006.

[2] K. Zhang, J. Yang, X. Yu, J. Zhang, and X. Wei, "Densities and viscosities for binary mixtures of poly(ethylene glycol) $400+$ dimethyl sulfoxide and poly(ethylene glycol) $600+$ water at different temperatures," Journal of Chemical and Engineering Data, vol. 56, no. 7, pp. 3083-3088, 2011.

[3] A. Ali, A. K. Nain, D. Chand, and R. Ahmad, "Viscosities and refractive indices of binary mixtures of dimethylsulphoxide with some aromatic hydrocarbons at different temperatures: an experimental and theoretical study," Journal of the Chinese Chemical Society, vol. 53, no. 3, pp. 531-543, 2006.

[4] J. A. Riddick, W. B. Bunger, and T. K. Sakano, Organic Solvents: Physical Properties and Methods of Purifications, vol. 2 of Techniques of Chemistry, John Wiley \& Sons, New York, NY, USA, 1986.

[5] V. K. Rattan, S. Kapoor, and K. Tochigi, "Viscosities and densities of binary mixtures of toluene with acetic acid and propionic acid at $(293.15,303.15,313.15$, and 323.15) K,' Journal of Chemical and Engineering Data, vol. 47, no. 5, pp. 1182-1184, 2002.

[6] R. A. McAllister, "The viscosity of liquid mixtures," AIChE Journal, vol. 6, pp. 427-431, 1960.

[7] L. Grunberg and A. H. Nissan, "The energies of vaporisation, viscosity and cohesion and the structure of liquids," Transactions of the Faraday Society, vol. 45, pp. 125-137, 1949.

[8] G. C. Benson and O. Kiyohara, "Evaluation of excess isentropic compressibilities and isochoric heat capacities," The Journal of Chemical Thermodynamics, vol. 11, no. 11, pp. 1061-1064, 1979.

[9] G. Douhéret, M. I. Davis, I. J. Fjellanger, and H. Høiland, "Ultrasonic speeds and volumetric properties of binary mixtures of water with poly(ethylene glycol)s at $298.15 \mathrm{~K}$," Journal of the Chemical Society - Faraday Transactions, vol. 93, no. 10, pp. 1943-1949, 1997.

[10] A. J. Treszczanowicz, O. Kiyohara, and G. C. Benson, "Excess volumes for $n$-alkanols $+n$-alkanes IV. Binary mixtures of decan-1-ol +n-pentane, + n-hexane, + n-octane, + n-decane, and $+n$-hexadecane," The Journal of Chemical Thermodynamics, vol. 13, no. 3, pp. 253-260, 1981.

[11] A. H. Roux and J. E. Desnoyers, "Association models for alcohol-water mixtures," Journal of Proceedings of the Indian Academy of Sciences: Chemical Sciences, vol. 98, no. 5-6, pp. 435-451. 

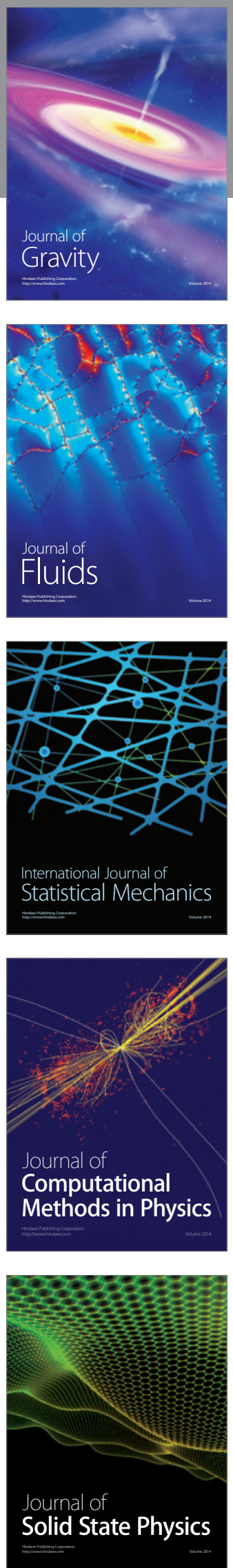

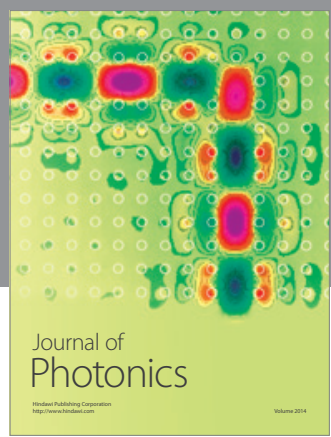

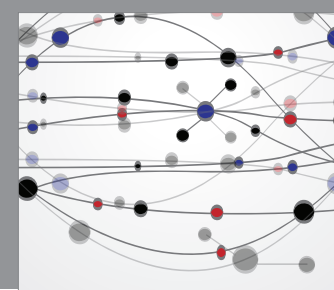

The Scientific World Journal

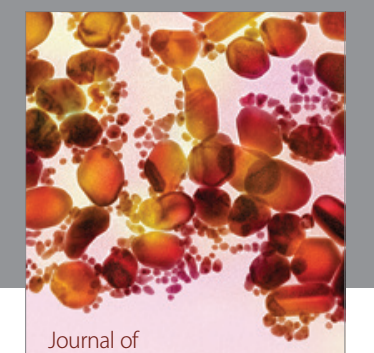

Soft Matter
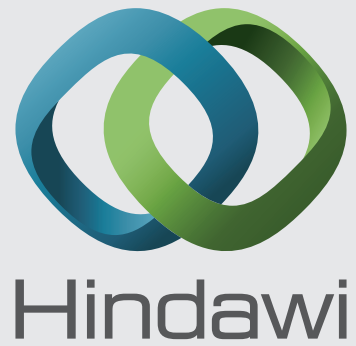

Submit your manuscripts at

http://www.hindawi.com
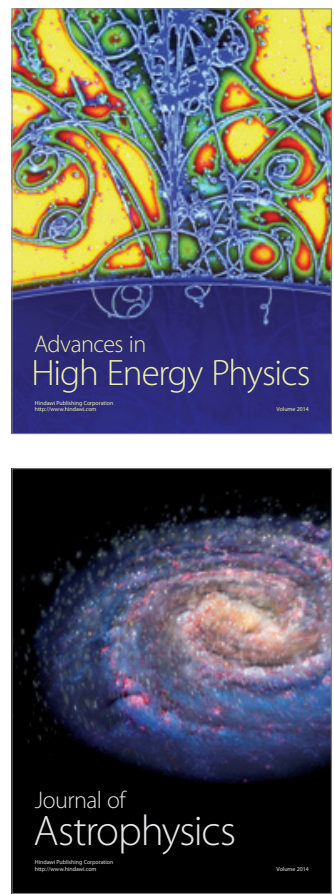
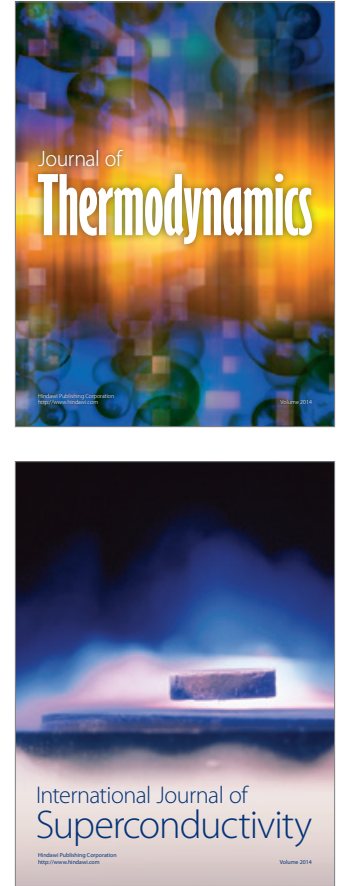
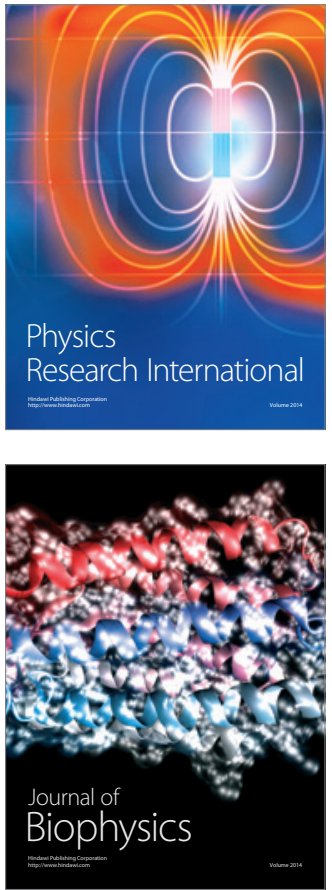
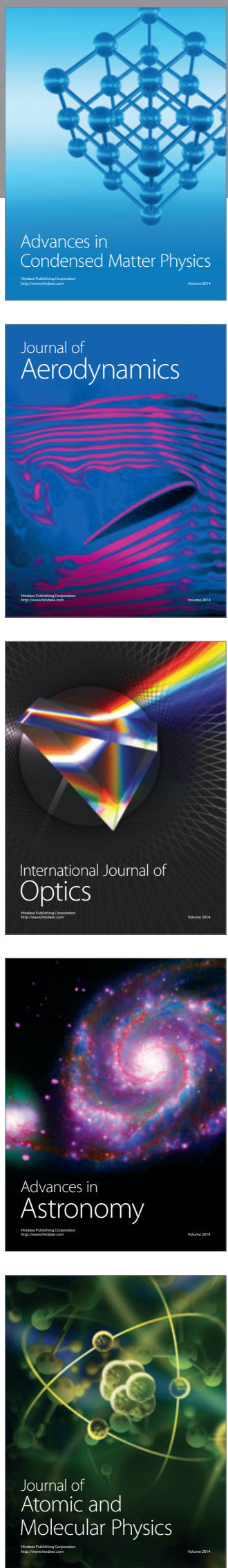